\title{
POLITECNICO
}

MILANO 1863

RE.PUBLIC@POLIMI

Research Publications at Politecnico di Milano

This is the accepted version of:

M. Nugnes, C. Colombo, M. Tipaldi

A System Engineering Tool for the Optimisation of a Gnss Constellation Design

in: 5th IEEE International Workshop on Metrology for AeroSpace (MetroAeroSpace), Rome, Italy, 20-22 June 2018, ISBN: 9781538624746, p. 300-305

doi:10.1109/MetroAeroSpace.2018.8453600

The final publication is available at https://doi.org/10.1109/MetroAeroSpace.2018.8453600

When citing this work, cite the original published paper.

Permanent link to this version

http://hdl.handle.net/11311/1076438 


\section{A System Engineering Tool for the Optimisation of a GNSS Constellation Design}

\author{
Marco Nugnes \\ Department of Aerospace Science \\ Politecnico di Milano \\ Milano, Italy \\ marco.nugnes@mail.polimi.it
}

\author{
Camilla Colombo \\ Department of Aerospace Science \\ Politecnico di Milano \\ Milano, Italy \\ camilla.colombo@polimi.it
}

\author{
Massimo Tipaldi \\ Department of Engineering \\ University of Sannio \\ Benevento, Italy \\ mtipaldi@unisannio.it
}

\begin{abstract}
This paper introduces a system engineering tool for the optimisation of a generic GNSS constellation design by using Galileo as reference. The optimisation is performed by taking into account both the percentage of global coverage and the accuracy in the position determination. The tool executes the optimisation not only for nominal and ideal cases, but also for off-nominal configurations involving catastrophic or transient failures of the constellation satellites. The analysis of the GNSS robustness to failures changes considerably the number of satellites to be used per plane with respect to the original configuration designed in nominal conditions.
\end{abstract}

Index Terms-GNSS, Galileo satellites, constellation design, space engineering tool, Earth coverage.

\section{INTRODUCTION}

The use of GNSS systems is becoming one of the most adopted solutions not only for Earth navigation missions but also for Earth observations of the atmosphere by using remote sensing techniques (for instance, the study of the ionosphere Total Electron Content [1] and the global ocean altimetry [2]). A GNSS is a complex system which consists of 3 main parts:

- The space segment, a group of navigation satellites in orbit around the Earth placed in a precise orbit positioning according to a strategy to get a minimum requirement of global coverage.

- The ground segment, responsible for managing the constellation of navigation satellites, controlling core functions of the navigation missions as well as determining and disseminating the integrity information.

- The user segment, a receiver converting the navigation signal of the satellites in position and velocity at a specific time.

GNSS systems offer positioning services with a high level of availability (more than $99.5 \%$ ). These applications arouse interest for all the fields where the precise positioning is required. Indeed, in the last years GNSS systems are focusing on the transportation sector to increase the security of the payload on board. The consequence is the fulfilment of tight requirements about the global coverage and the accuracy on the position determination. The only international entity providing the requirements for the GNSS systems up to now is the ICAO (International Civil Aviation Organisation) but in the next years also the rail and maritime organisations will take part in the program.

For these reasons it is important to provide a method to optimise the GNSS design to achieve the highest performance. In this field, Sünderhauf et al. [3] developed a method to optimise them by mitigating the multipath effect and ensuring a better localisation of a generic user in the big cities where the multipath phenomenon is more present. Another way to look at the problem is to focus on the ground segment, as Coulot et al. [4] state in their article where an optimisation of the reference stations network is performed to have a better communication with the space segment. It is important to take into account also the interactions of the navigation signal with the atmosphere and this is what Pan et al. [5] focus on by optimising the positioning performance in harsh environments considering a double-difference (DD) model as solution to the problem.

Ashkenazi et al. [6] introduces the basic theoretical principles of the design of optimal satellite orbits in terms of satellite availability, coverage, accuracy and integrity. Instead, Tadic et al. [7] describes the design of software tools for GNSS performance analysis and signal propagation simulation builtin a web portal comparing the results with existing GNSS performance analysis tool (GISMO, EUROMON) and GNSS propagator simulators (WinProp, NavTK, Polaris).

In all the previous papers, an optimisation of the GNSS performance is carried out improving some specific aspects. To the best of our knowledge, a computer-aided approach (including also failure scenarios) for the overall design of the GNSS space segment in terms of number of satellites and their configuration is new in the literature. This paper introduces a GNSS tool that can be exploited for the optimisation of the constellation configuration not only considering a nominal behaviour, but also in presence of catastrophic [8] (the satellite cannot be recovered unless it is replaced by another one) or recoverable failures. The analysis of the GNSS robustness to failures results in the number of operative satellites and spare satellites per plane satisfying the requirements about the global coverage and accuracy in the position determination. The paper is organised as follows. In this section an overview about GNSS systems and their design optimisation is presented. In section II the GNSS tool is described together with its 
embedded models. Section III introduces some examples of usage to validate the tool by using Galileo as reference, while section IV shows the outcome of the optimisation. Finally, section $\mathrm{V}$ concludes the paper.

\section{GNSS TOOL Functional Model}

This section describes the architecture of the tool starting from the functional decomposition up to the basic models embedded in the tool in order to understand how it works. Hence, the tool is named G-CAT which stands for GNSS Coverage Analysis Tool. G-CAT consists of three main blocks as shown in Fig. 1: the space segment, the coverage part and the interaction with the atmosphere.

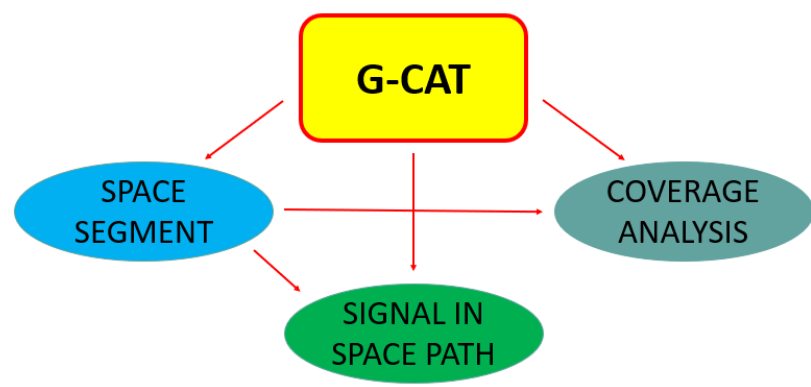

Fig. 1. G-CAT architecture.

\section{A. Space Segment}

The space segment block models the GNSS constellation and in particular the in-orbit satellites both from an orbital and attitude point of view. This block consists of an orbit propagator which must take as input the initial conditions of the GNSS constellation given in terms of orbital elements, and an attitude propagator for calculating the attitude of the spacecraft and in particular way the line of sight of the navigation antenna. Then, all the functions computing the instantaneous access area, which is the locus of points in view of a satellite at a particular instant from a given position, are part of this block. It must be underlined that the propagation of the satellites' orbits is decoupled from the attitude. Therefore, for the orbit propagation, each satellite is assumed to be a dot mass coincident with its centre of mass. The computation of the instantaneous access area, conversely, can couple the orbit and the attitude data.

\section{B. Signal in Space Path}

The second main block is related to the interaction of the navigation signal with the atmosphere. All the functions implemented in this block are not sufficiently accurate. However, they represent first-order methods to model both the troposphere and the ionosphere. Indeed, whenever an electromagnetic wave propagates through the atmosphere there is the presence of refraction, scintillation and multipath. Only refraction is considered in G-CAT. Multipath is the reception of the same signal sent at an earlier time instant caused by the ground reflection of that signal. In order to better represent all the interactions, statistics must be introduced. Therefore, G-CAT gives as output some figures about the tropospheric and ionospheric delay together with the link budget starting from the properties of the navigation signal.

\section{Earth Coverage Analysis}

The third block is related to the coverage analysis which gives as output the performance of the GNSS. It consists of all the functions that take as input the instantaneous access area of each satellite of the GNSS constellation and count for each point on the Earth the number of satellites in view. GCAT gives the possibility to compute the instantaneous access area modelling the Earth's shape both as a sphere and an oblate ellipsoid of rotation according to the WGS-84 (World Geodetic System 1984) representation [9]. From the number of satellites in view for each point on the Earth's surface, three coverage indices are identified to measure the performance of the GNSS system:

- "Red coverage index", percentage of Earth's surface in view of less than 4 satellites.

- "Yellow coverage index", percentage of Earth's surface in view of exactly 4 satellites.

- "Green coverage index", percentage of Earth's surface in view of more than 4 satellites.

The previous indices have been defined taking into account that 4 measurements of the satellite-receiver distance are the minimum number in order to get the position of the receiver's location.

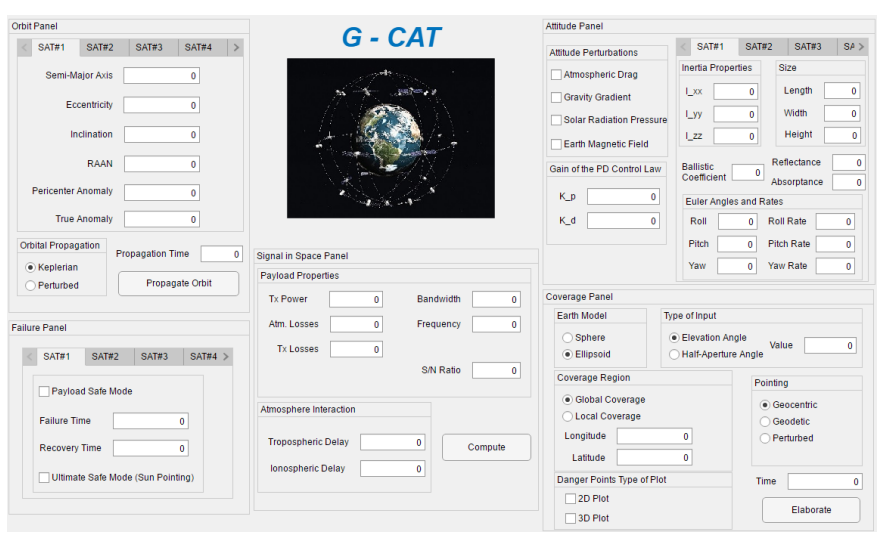

Fig. 2. G-CAT graphical interface.

\section{G-CAT VAlidation, Features And Us AGE}

This section describes briefly the features of the G-CAT tool starting from the user-friendly graphical interface shown in Fig. 2. In particular, some test cases are presented to show the outputs and the results offered by the tool.

The first case considers the nominal case of Galileo constellation to validate that the tool computes correctly its global coverage. The second case introduces the presence of a catastrophic failure to verify the behaviour of the constellation and, finally, a third case underlines the difference with respect to the previous case when another pointing is analysed. 


\section{A. Nominal Case}

Galileo is a 24/3/1 Walker Delta pattern constellation [10]. This means that there are 24 satellites distributed in 3 equally spaced planes, 8 satellites for each plane spaced of $45^{\circ}$ and a phasing angle between two adjacent planes of $15^{\circ}$. After inserting the orbital elements of the constellation, it is necessary to select the type of representation of the Earth and choose the input and the pointing.

- Ellipsoidal representation of the Earth.

- Minimum elevation angle equal to $5^{\circ}$.

- Geocentric pointing.

The outcome of this first test case is shown in Fig. 3.

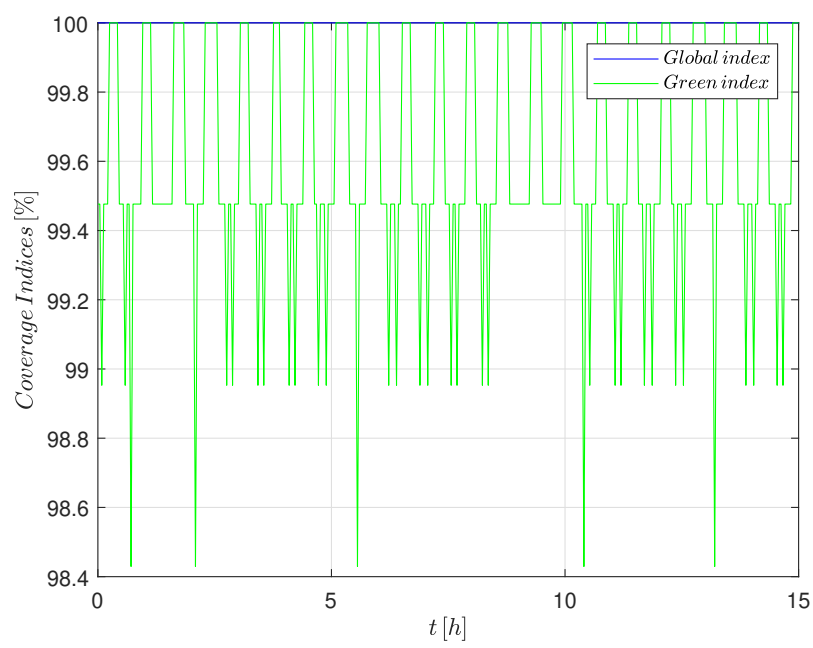

Fig. 3. Coverage indices for the nominal configuration.

The coverage is global and the "green index" highlights that the Earth's surface is in view of more than 4 satellites and this ensures also a high accuracy in the position determination.

\section{B. Failure Case \#1}

In the second example, an off-nominal configuration is analysed. In particular, a catastrophic failure of one satellite is considered. This time the type of input chosen is the halfaperture angle and the pointing is geodetic along the local vertical.

- Ellipsoidal representation of the Earth.

- Half-aperture angle equal to $12^{\circ}$.

- Geodetic pointing.

- \#1 satellite (first satellite of the first orbital plane) fails with no possibility to be recovered and without the substitution of the spare satellite.

As for this test case, the outcome is presented in terms of the critical points, that is to say the points that at least for one instant during the propagation of the constellation are totally uncovered. These critical points are underlined with red crosses as shown in Fig. 4 in such a way that the uncovered regions can be directly associated with the geographic representation.

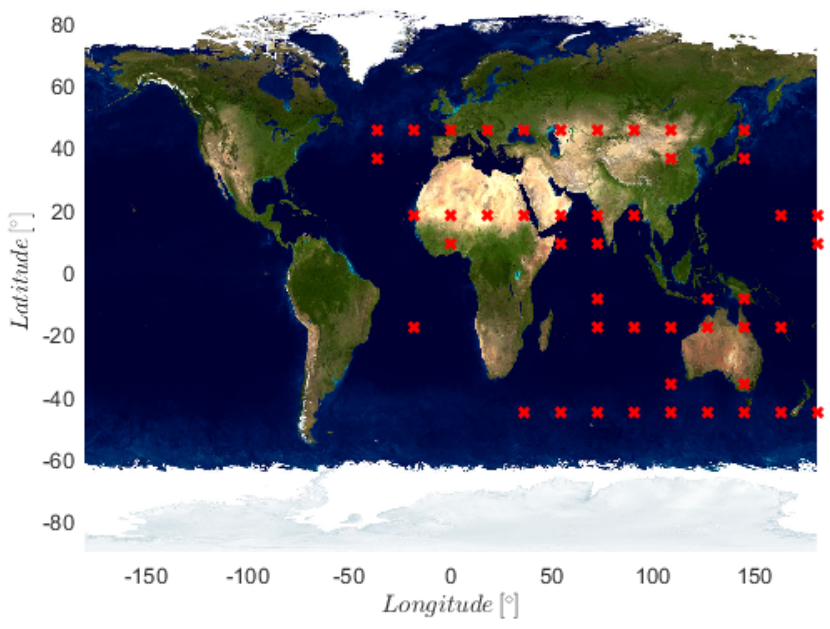

Fig. 4. Critical points in case of one satellite permanent failure.

\section{Failure Case \#2}

The last test case highlights the importance of considering a moving and perturbed direction for the line of sight of the navigation signal. The inputs for the analysis are the same of the previous example. The only change is in the type of pointing:

- Ellipsoidal representation of the Earth.

- Half-aperture angle equal to $12^{\circ}$.

- Perturbed pointing for all the satellites.

- \#1 Satellite fails.

Even if the result in terms of global coverage index is similar to the one obtained for the geodetic pointing, the situation changes if the map of the critical points is examined.

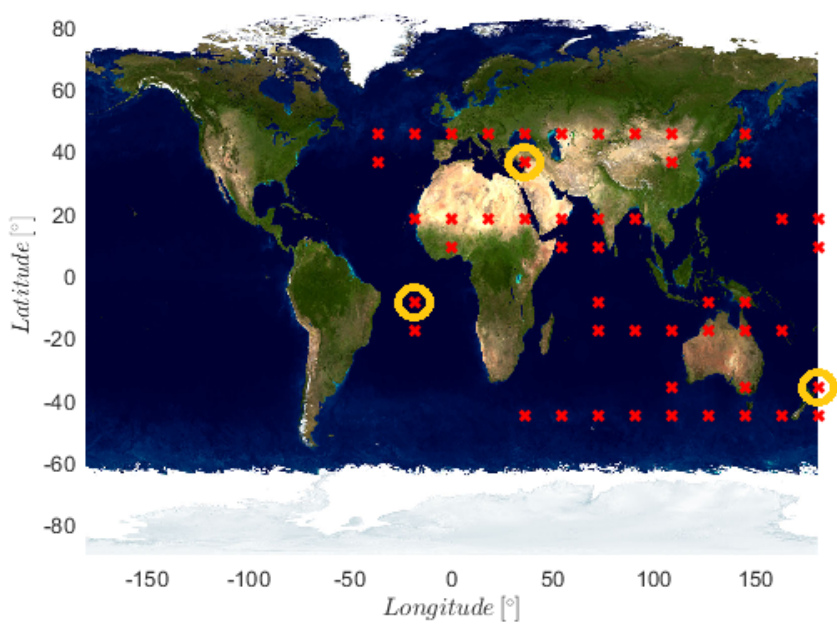

Fig. 5. Critical points in case of one satellite permanent failure and perturbed pointing.

The orange circles in Fig. 5 show the differences with respect to the previous case. There are some points in the 
perturbed case that are not present in the geodetic case and the other way around. So, even if from a global coverage percentage point of view the situation is almost the same, the covered and uncovered regions change. Moreover, the moving line of sight is a better representation than the simple fixed direction since the attitude perturbations are always acting on the spacecraft.

\section{Optimisation Process Applied to Galileo CONSTELlation}

This section is devoted to the computation of the optimal configuration for the Galileo constellation design keeping fixed the number of planes in which the different satellites can be distributed. Such optimisation procedure can be performed by processing the different configuration scenarios with G-CAT, storing the results obtained and, finally, creating a plot with the data stored. First, it is necessary to analyse which are the configurations for the constellation granting a global coverage at least in the nominal case since it is a requirement that must be satisfied in any case.

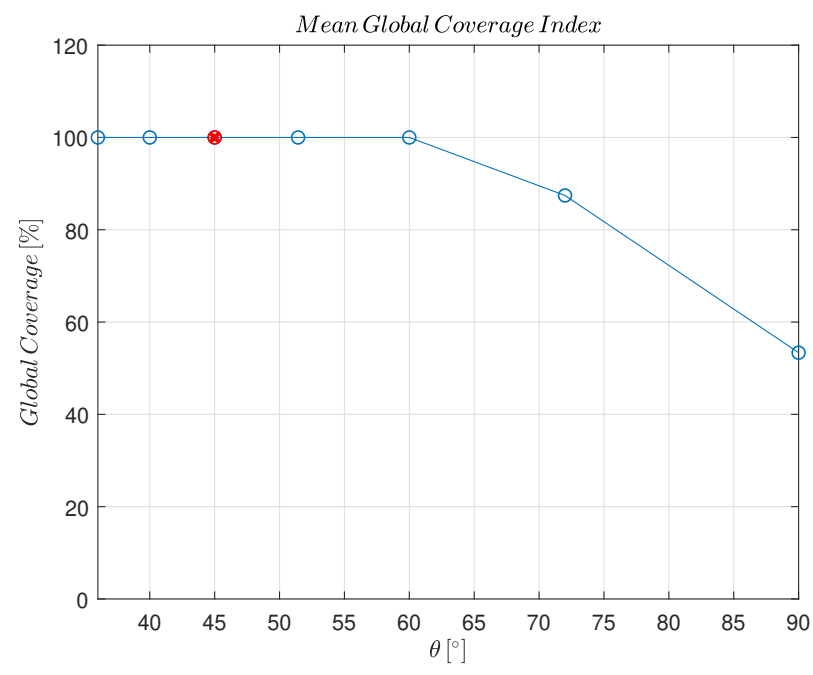

Fig. 6. Mean global coverage index for different constellation configurations.

In Fig. 6 this problem is analysed for 7 types of configurations. Each big dot in the plot represents a scenario analysed. On the abscissa axis the parameter $\theta$ represents the spacing between the satellites within the same orbital plane and so it uniquely identifies the number of total satellites of the constellation (from 4 satellites to 10 satellites for each plane). The surprising result is that the global coverage is obtained also for geometries containing 6 or 7 satellites for each plane. Taking into account that the Galileo nominal configuration includes 8 satellites for each plane, the natural question is:

Why is the actual constellation configuration involving 8 satellites when there are other cheaper configurations giving the same result?
In order to answer to this question, the off-nominal behaviour of the different configurations must be studied. In particular, 3 particular failure scenarios have been selected:

- Failure case 1: complete loss of \#1 satellite of the first plane.

- Failure case 2: complete loss of \#1 satellite of the first plane and \#2 satellite of the second plane.

- Failure case 3: mix of recoverable failures affecting \#1 and \#2 satellites of the first plane, \#3 satellite of the second plane and \#2 satellite of the third orbital plane.

The two parameters to be optimised are the global coverage index and the green coverage index.

\section{A. Global Coverage Optimisation}

The first parameter analysed is the global coverage representing the sum of the yellow and green coverage indices. The results of the global coverage optimisation are shown in Fig. 7 where on the $x$-axis the spacing between the satellites in the same orbital plane is shown, while on the $y$-axis there is the global coverage index associated for one revolution of the constellation.

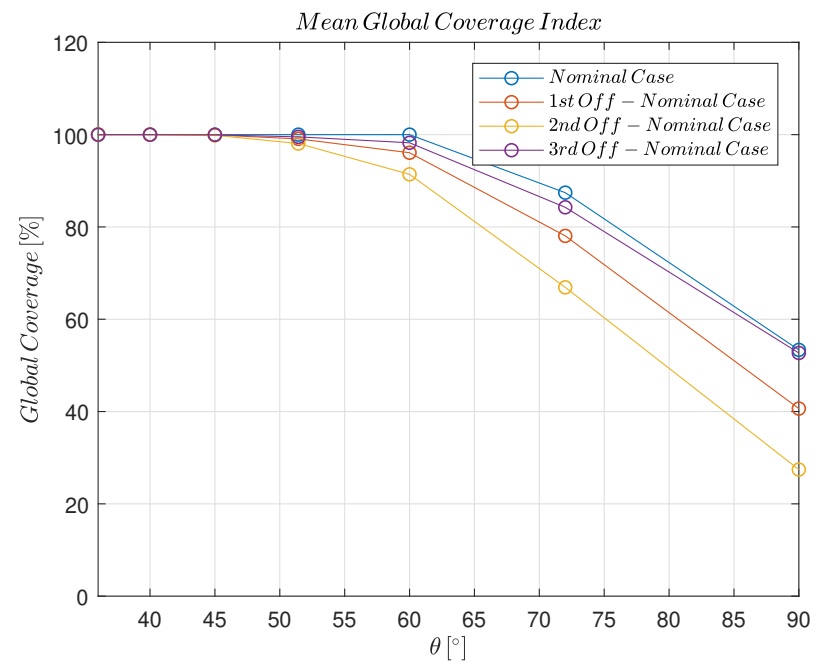

Fig. 7. Mean global coverage index for different cases.

It is clear that the higher the number of satellites for each plane is, the higher the robustness to these failures is. In particular, the constellation seems to be robust up to a value of $\theta=51.43^{\circ}$ corresponding to 7 satellites for each plane since the value of the global coverage index is near the maximum and starting from the a configuration involving 6 satellites for each plane, the decreasing slope becomes quite high.

\section{B. Accuracy Optimisation}

The other variable to be optimised is the green coverage index identifying the number of points on the Earth's surface in view of more than 4 satellites. Indeed, even if the minimum number of satellites to compute the position of a generic user is 4 , this does not imply that the result is good. The higher the number of measurements is, the better the accuracy and 
precision for user position can be achieved. Since the precision requirements for a GNSS are very tight (in the order of meters) this parameter cannot be neglected.

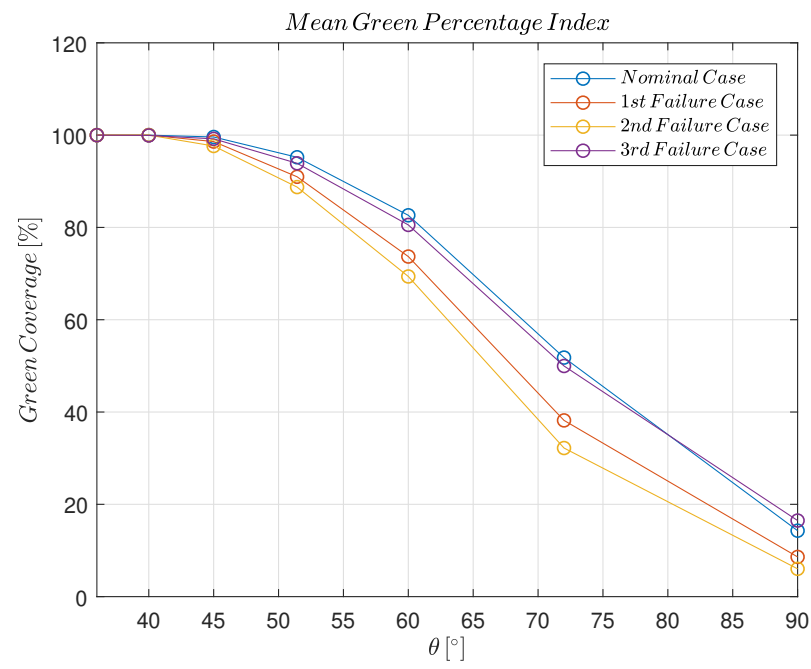

Fig. 8. Mean green percentage index for different constellation configurations.

In Fig. 8 it is clear that the best configuration between 7 and 8 satellites for each plane is the latter since the slope of the green index becomes relevant starting from the geometry involving 7 satellites for each plane. For a GNSS constellation like Galileo, where the coverage must be global and the accuracy of the user position is very high, the only possibility is to use at least a configuration with 8 satellites for each plane which is the actual one. The surprising result is that Galileo original nominal configuration involved 9 operating satellites for each orbital plane. Therefore, the reduction in the nominal condition from 9 satellites to 8 satellites for each plane seems to be a good choice because the coverage performance is not so affected from one side while the cost of the project considerably decreases.

\section{COnClusions And Future Work}

In this paper a system engineering tool for the optimisation of a generic GNSS design is presented. The tool uses as input a given configuration in terms of orbital planes and takes into account as parameters for the optimisation the global coverage percentage of the GNSS constellation and the accuracy of the position determination both in nominal conditions and in presence of catastrophic or recoverable failures. The optimisation procedure has been validated by considering the Galileo constellation as well as its design evolution and history.

As for future work, the G-CAT tool can be empowered by including in the optimisation process the number of the orbital planes where the satellites are to be placed. This way, we can set up the constellation geometry fulfilling the global coverage and accuracy requirements in both nominal and offnominal conditions and thoroughly support the design of the space mission.

\section{ACKNOWLEDGMENT}

This work was supported by Politecnico di Milano under the COMPASS project (Control for Orbit Manoeuvring through Perturbations for Application to Space Systems), grant agreement 679086 .

\section{REFERENCES}

[1] E. Musicò, C. Cesaroni, L. Spogli, J. P. M. Boncori, G. De Franceschi, R. Seu, "The Total Electron Content From InSAR and GNSS: A Midlatitude Study", IEEE Journal of Selected Topics in Applied Earth Observations and Remote Sensing, vol. 11, May 2018, pp. 1725-1733.

[2] J. Mashburn, P. Axelrad, S. T. Lowe, K. M. Larson, "Global Ocean Altimetry With GNSS Reflections From TechDemoSat-1", IEEE Transactions on Geoscience and Remote Sensing, April 2018, pp. 1-10.

[3] N. Sünderhauf and M. Obst and G. Wanielik and P. Protzel, "Multipath mitigation in GNSS-based localization using robust optimization", 2012 IEEE Intelligent Vehicles Symposium, June 2012, pp. 784-789.

[4] D. Coulot, P. Rebischung, A. Pollet, L. Grondin, G. Collot, "Global optimization of GNSS station reference networks", GPS Solutions Journal, vol. 19, October 2015, pp. 569-577.

[5] S. Pan, X. Meng, W. Gao, S. Wang, A. Dodson, "A New Approach for Optimising GNSS Positioning Performance in Harsh Observation Environments", Journal of Navigation, vol. 67, November 2014, pp. 1029-1048.

[6] V. Ashkenazi, W. Chen, W. Y. Ochieng, C. J. Hill, T. Moore, "Design Tools for GNSS", IEE Colloquium on Implementation of GNSS, November 1995 .

[7] S. Tadic, G. Moura, T. Trichaud, "Design of GNSS Performance Analysis and Simulation Tools in the "GAPFILLER" Web Portal", 2013 21st Telecommunications Forum Telfor (TELFOR), November 2013.

[8] ECSS-Q-ST-30-02C: Space product assurance - Failure modes, effects (and criticality) analysis (FMEA/FMECA), European Cooperation for Space Standardization, 2009.

[9] David A. Vallado, Fundamentals of Astrodynamics and Applications, 4th edition, Space Technology Library, California, 2013, pp. 131-133.

[10] European Global Navigation Satellite Systems Agency, EGNOS Open Service (OS) Service Definition Document, rev. 2.3, 2017, pp. 2-5. 